\title{
Magnetized Structures in Sgr A West
}

\author{
D.K.Aitken \\ University College, University of New South Wales \\ Australian Defence Force Academy \\ Campbell, ACT 2600 \\ Australia
}

\begin{abstract}
Spectropolarimetric studies of Sgr A West at $10 \mu \mathrm{m}$ reveal intrinsically polarized emission from the northern arm. The polarization, of amount $7-8 \%$, is attributed to emission from dust grains which are aligned by a magnetic field directed along the line of the northern arm. Along the eastern arm the polarization is smaller and indicates either a weaker field or one directed closer to the line of sight.

Polarized emission has also been observed at $100 \mu \mathrm{m}$ from aligned grains in the ring of molecular gas which apparently encircles Sgr A. The aligning magnetic field lies in the plane of the ring and is probably toroidal. The correspondence between the polarization position angles at 10 and $100 \mu \mathrm{m}$ suggests a model in which the northern arm is a disrupted filament from the molecular ring.

While the uncertain magnetic properties of grains prevent a reliable direct estimate of field strength, circumstantial evidence suggests it is larger than elsewhere in the Galaxy and possibly large enough to influence the dynamics of the region.
\end{abstract}

\section{Introduction}

The discovery of the polarization of starlight (Hiltner, 1949; Hall, 1949) showed that the dust grains responsible for the interstellar extinction are capable of alignment. While there has been to date no completely satifactory grain alignment mechanism proposed, it is clear that the alignment direction is determined by the ambient magnetic field. Working on this basis alone, Chandrasekhar and Fermi (1953) attributed the scatter of field directions in the Galactic plane to Alfvén waves driven by turbulent motions and derived a Galactic field strength $\simeq 7 \mu$ gauss, a value which does not depend on details of the alignment mechanism or the physical and chemical properties of the dust grains. This field magnitude has since been supported by studies of Faraday rotation (Gardner, Morris and Whiteoak, 1969) and the dispersion of pulsar signals (Manchester, 1974). 


\section{Polarized emission from the Galactic centre:}

\section{1 the mid infrared}

In the infrared, polarization has been observed in heavily obscured sources and in many cases is attributable to dichroic absorption by aligned grains. Polarized emission from aligned grains has also been observed in a number of sources, and in particular the $10 \mu \mathrm{m}$ polarization observed towards the Galactic Centre has been variously attributed to absorption, emission and combinations of these (Knacke and Capps, 1977; Lebofsky et al, 1982; Aitken et al, 1986)

Separation of the polarization due to absorption by aligned grains from that due to emission is facilitated by spectropolarimetric observations. Silicate materials have a resonance near $10 \mu \mathrm{m}$, and in absorption this introduces a polarization peak at a slightly longer wavelength (e.g. Martin, 1975), with relatively small polarization at 8 and $13 \mu \mathrm{m}$. Polarized emission from the same grains, however, exhibits a much weaker spectral dependence and is "tilde" shaped with a weak minimum near $9 \mu \mathrm{m}$ and broad maximum in the 12 to $13 \mu \mathrm{m}$ region; the general form of polarized emission is a guide to the band strength of the resonance and the degree of short term order in the grain material (Martin, 1975). When a source emitting polarized radiation suffers extinction by a medium of aligned grains this is often revealed by a change of the position angle of polarization through the resonance; the position angle near the band centre being pulled towards that of absorptive polarization and at the edges of the band tending to that of the emission component.

Such a situation is well illustrated by the observations of Sgr A (Aitken et al, 1986). Here there is polarized emission from aligned dust grains in $\mathrm{Sgr} \mathrm{A}$, and the long path in the diffuse interstellar medium to the Galactic Centre overlays this with absorptive polarization, introducing a change of the position angle of polarization with wavelength, especially close to the band centre. One of the sources, IRS3, which has no radio counterpart or infrared fine structure lines, shows no emissive component while the ionized filaments, particularly those in the northern arm, have strong intrinsic polarizations and show spatial variations in their position angles.

The interest here is in the intrinsic polarization and the magnetic field strength and orientation implied, although the absorptive polarization is important in its own right as it represents a long path length through the diffuse interstellar medium. To unfold the interstellar component of polarization from the observations requires a knowledge of its polarization spectrum, and in principle this could be provided by the observations of IRS3 itself. In practise much higher quality data exist for a number of heavily obscured sources in molecular clouds, the BN object in Orion being a particularly well studied case. This has been used to approximate the interstellar component (Aitken et al, 1986) by scaling its peak polarization to the IRS3 peak value and adopting the IRS3 position angle of $0^{\circ}$. When this is applied to the various sources in Sgr A the implicit assumption is, of course, that the interstellar polarization is constant across the region, of extent $\sim 1 \operatorname{arcmin}$. Fortunately this interstellar contribution to polarization is small at $12-13 \mu \mathrm{m}$ and, for the strongly polarized sources in the northern arm at least, the derived polarization position angle differs only 
slightly $\left(<7^{\circ}\right)$ from the observed position angle at $12.5 \mu \mathrm{m}$; thus precise knowledge of the form of the interstellar polarization curve is not critical. For the Galactic Centre a simple equivalent to this procedure for the $12-13 \mu \mathrm{m}$ region is to subtract $1.8 \%$ from the Stokes parameter $Q$ for the data.

For polarized emission from aligned grains the polarization fraction is related to the projection on the plane of the sky of the degree of grain alignment while the $E$ vector direction is orthogonal to the projected direction of the aligning magnetic field. Fig 1 shows the implied field directions and alignments determined from observations using the UCL spectropolarimeter on the UKIRT and the AAT (from Aitken et al, 1986; Smith, 1988; and in preparation) superimposed on a grey scale map of part of the Sgr A complex obtained using the same instrument on the AAT with an aperture of 2.1 arcsec diameter (Smith, 1988 and poster at this symposium). It is clear that the magnetic field closely links the sources in the northern arm up to IRS8, and curves into the central ridge southwest of IRS1. There is also a correlation between the field directions in the east-west bar and the local details of the morphology: for instance the field is closely aligned with the elongation of IRS2 and also follows the trend of the fine structure of the radio continuum maps (e.g. Morris and Yusef-Zadeh, 1987). In the northern arm the polarization is large (7-8 \%) and uniform, and at IRS1 independent of beam size over a range 2.1-5.6 arcsec. The polarization implies stronger alignment than in any other source so far observed (the $12.5 \%$ observed in the BN object is due to absorption and depends on optical depth; $\mathrm{p} / \tau=12.5 / 3.3 \simeq 4 \%$ ) and this has implications regarding the field strength and the alignment mechanism which are discussed later. The nearly uniform polarization also suggests that any curvature of the northern arm normal to the plane of the sky is $<30^{\circ}$, while in the east-west bar the lower polarization may simply be the result of projection.

\section{2 the far infrared}

Polarization at the few \% level has also been observed by Hildebrand and co-workers at $100 \mu \mathrm{m}$ (Werner et al, 1988; Hildebrand et al, in preparation) at a number of positions along the dust ring surrounding Sgr A (Becklin, Gatley and Werner, 1982; Lester et al, 1987; Davidson et al, 1988). These observations have been made with aperture sizes of 55 and 40 arcsec using the University of Chicago Airborne Polarimeter on the Kuiper Airborne Observatory and extend to positions $~ 75$ arcsec north and south of the centre. The polarization reaches a maximum of almost $3 \%$ on the northern infrared peak of the dust ring and declines on either side of this. At this wavelength optical depths are small and the natural inference is that the polarization is due to emission from aligned grains; the implied field directions follow closely the observed line of dust emission. At the central position the aperture includes the ionized filaments of the northern arm, central ridge and east-west bar; estimates of the polarized intensity at this position indicate that a substantial part of it may arise from the same grains which supply the $10 \mu \mathrm{m}$ polarization. However for the positions offset from the centre the polarization must arise from grains in the predominantly neutral dust ring and imply a magnetic field within the ring, aligned in its plane and presumably toroidal. Again the polarizations are stronger than elsewhere observed in this wavelength range, and have implications regarding the field strength in the ring and the alignment 


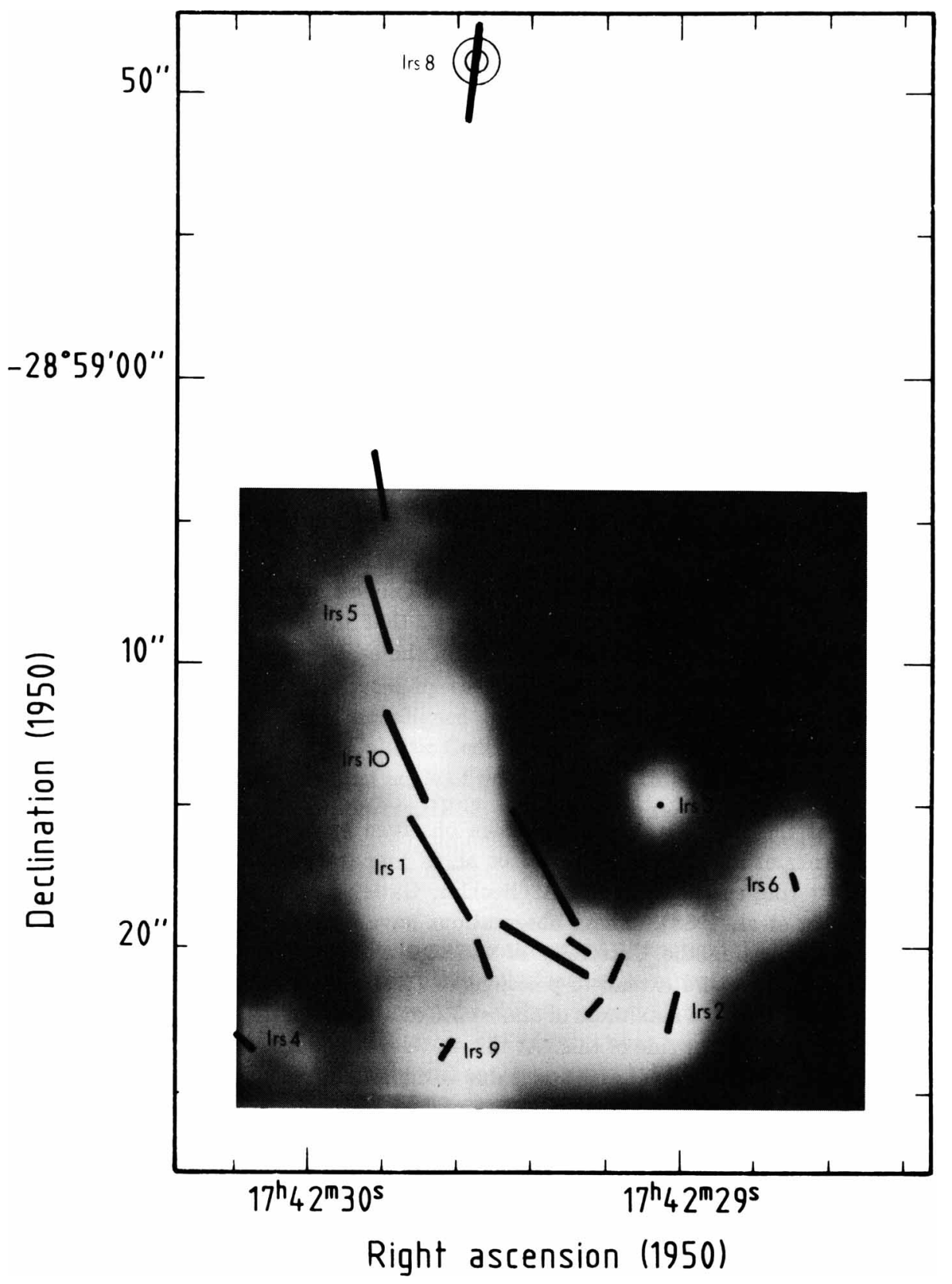

Figure 1: Magnetic field directions in Sgr A. See text for details. This material is being prepared for publication in a more complete form. 
mechanism in neutral regions.

\section{3 the near infrared}

While the absorptive interstellar component of polarization is correspondingly important at short wavelengths, nevertheless indications of an emissive component in a number of Sgr A sources have been found by Knacke and Capps (1977), Lebofsky et al (1982) and Bailey et al (1984). These data have been re-evaluated by Aitken et al (1986) in the light of a better understanding of the role of extinction and clearly show polarized emission in IRS1, IRS5 and IRS10 at the $6 \%$ level at $3.5 \mu \mathrm{m}$. Here the polarization must arise from a population of much hotter grains than those which give the $10 \mu$ polarization, but the position angles are similar and suggest that the same field orients these populations. Some of the implications of the alignment of such hot grains $(T \gtrsim 750)$ are discussed in the next nextion.

\section{The alignment mechanism and the field strength}

Due to mechanical hysteresis and, more importantly, the Barnett effect (e.g. Landau and Lifshitz, 1960) dust grains will spin about an axis normal to their long dimension. Again due to the Barnett effect their spins will precess about the ambient magnetic field at a rate much faster than any other time constant of grain motion. Because of this overiding effect the orientation of grain alignment will always be determined by the ambient field, irrespective of the actual mechanism of alignment. Nevertheless, in the case of the Galactic centre it does seem that the prime aligning mechanism is indeed the magnetic field (Aitken et al, 1986) and that in the $10 \mu \mathrm{m}$ region the classical Davis-Greenstein (DG) mechanism (1951) is capable of producing the alignment required by the observed polarizations in these ionized regions. Assuming purely paramagnetic grains the field strength required at the gas densities envisaged in the northern $\operatorname{arm}\left(\mathrm{n}_{e} \sim 10^{5} \mathrm{~cm}^{-3}\right)$ is $\gtrsim 10$ mgauss.

The straightforward DG mechanism has always overestimated the field strength by a factor between 3-10 when compared with other determinations and it is difficult to see how it can produce alignment at all in dense neutral regions where the grain and gas temperatures should be tightly coupled. An essential requirement of magnetic alignment by relaxation is a difference between the internal temperature and the spin temperature of the grains, and this has led to the proposal that in many cases grain spin can be suprathermal (Purcell, 1979), that is to say greater than expected from equipartion with the ambient gas. Plausible spin-up mechanisms include hydrogen molecule recombination and ejection from specific sites on grains and a similarly site-specific photo-electric effect. Suprathermal spin makes magnetic alignment possible but does not speed it up and an additional requirement frequently invoked is to assume that the grains which are aligned contain superparamagnetic (SPM) inclusions (eg Mathis, 1986) in order that magnetic relaxation can take place in times short compared to the disorientating effects of gas collisions and changing surface properties of grains. SPM inclusions can account for the field discrepancies noted earlier but a corollary is also that the alignment gives only a rough indication of the field strength. 
The large polarized emission observed in the Galactic centre over a wide range of wavelengths and which is almost constant along the northern arm and independent of the small scale structure suggests not only that the fields are much stronger here than elsewhere, but also that alignment is complete or nearly so. If this postulate is accepted then several inferences follow: (a) Suprathermal grain spin is now necessary in the northern arm as well as in the dust ring; in this ionized environment photoelectric effect from preferred sites would be a more likely mechanism for spin-up than hydrogen recombination on warm grains and alignment of grains with $\mathrm{T} \gtrsim 750 \mathrm{~K}$ almost certainly requires some sort of suprathermal process. (b) The grains need not be highly elongated or flattened and aspect ratios of only $\simeq 1.2$ can produce the observed polarization. (c) Estimates of field strength are in principle lower limits, although as we have seen the value of this limit is poorly defined. Nevertheless the apparent alignment saturation in a variety of conditions (temperatures over a range 50 to $\gtrsim 750 \mathrm{~K}$ and in both and neutral and ionized media) are circumstantial evidence that the field is much larger than the minimum value and probably exceeds the DG value of 10 mgauss.

The Chandrasekhar and Fermi (1953) arguments relate the field strength through the Alfvén velocity to observed turbulent velocities and spatial structure. With turbulent velocities $\sim 10 \mathrm{~km} \mathrm{~s}^{-1}$ the fields would become incoherent within the observing apertures on time scales < few thousand years unless the field strengths are in excess of 1-2 mgauss; this would appear to place a lower limit on the field strength in the northern arm. Applying the same arguments to the uniformity observed over the $\sim$ parsec extent of the arm yields fields an order of magnitude stronger than this; however here other dynamical effects, such as tidal stretching may provide the mechanism for the uniformity.

(d) Finally the lower polarizations seen in the east-west bar imply a field which is oriented $\simeq 60^{\circ}$ to the plane of the sky, and a corresponding tilt to the bar itself.

Thus while a reliable direct estimate of the field strength from the polarization is not possible, circumstantial evidence suggests the field is strong and in the range 5-20 mgauss. The field energy is then greater than the thermal and turbulent gas energy and not negligibly less than the kinetic energy of mass motions, and may influence these.

\section{Conclusions}

Observations of polarized emission from aligned dust grains over a range of wavelengths and positions in Sgr A lead to the following conclusions.

1. A magnetic field links the sources in the northern arm from IRS 8 to IRS1 and curves into the central ridge southwest of IRS1.

2. The dust ring encircling Sgr A contains a field lying in the plane of the ring and probably toroidal within the ring.

3. In the east-west bar the field orientations correlate with local features in the infrared and radio structure. 
4. Both in the northern arm and the neutral disc the grain alignments are stronger than seen in any other sources. This, and the spatial uniformity and apparent independence of grain temperature suggest that alignment is complete, or nearly so. In spite of the uncertainties in estimating field strengths from the polarimetric data this provides circumstantial evidence that the fields are at least as strong as the 10 mgauss predicted by standard Davis-Greenstein theory.

5. Assuming similar alignment throughout the region the fields in the east-west bar and presumably the bar itself are inclined at $\simeq 60^{\circ}$ to the plane of the sky.

\section{REFERENCES}

Aitken, D.K., Roche, P.F., Bailey, J.A., Briggs, G.P., Hough, J.H. \& Thomas, J.A., 1986. Mon. Not. R. Astr. Soc., $218,363$.

Bailey, J., Hough, J.H. \& Axon, D.J., 1984. Mon. Not. R. Astr. Soc., 206, 661.

Becklin, E.E., Gatley, I. \& Werner, M.N., 1982. Ap. J., 258, 135.

Chandrasekhar, S. \& Fermi, E., 1953. Ap. J., 118, 113

Davidson, Harvey, Lester, Morris \& Werner, 1988. in preparation.

Davis, L. \& Greenstein, J.L., 1951. Ap. J., 114, 206.

Gardner, F.F., Morris, D. \& Whiteoak, J.B., 1969. Aust. J. Phys., 22, 813.

Hall, J.S., 1949. Science, 109, 166.

Hildebrand, R.H., 1988. to appear in IAU Symp. 135.

Hiltner, W.A., 1949. Ap. J., 109, 471.

Landau, L.D. \& Lifshitz, E.M., 1960. Electrodynamics of Continuous Media, Reading, M.A.: Addison-Wesley, 144.

Knacke, R.F. \& Capps, R.W., 1977. Ap. J., 261, 271.

Lebofsky, M.J., Rieke, G.H., Deshpande, M.R. \& Kemp, J.C., 1982. Ap. J., 263, 672.

Lester, D.F., Joy, M., Harvey, P.M. \& Ellis, H.B., 1987. The Galactic Center, ed. Backer, D.C., AIP Conf. Proc. 155, 138.

Manchester, R.N., 1974. Ap. J., 188, 637.

Martin, P.G., 1975. Ap. J., 202, 393.

Mathis, J.S., 1986. Ap. J., 308, 281

Morris, M. \& Yusef-Zadeh, F., 1987. The Galactic Center, ed. Backer, D.C., AIP Conf. Proc. 155, 127. 
Purcell, E.M., 1979. Ap. J., 231, 404.

Smith, C.H., 1988. Thesis., University of Melbourne.

Werner, M.W., Davidson, J.A., Hildebrand, R.H., Morris, M.R., Novak, G. \& Platt, S.R., 1988 submitted to $A p$. J.. 